\title{
Photothermal Killing of Methicillin-Resistant Staphylococcus aureus by Bacteria-Targeted Polydopamine Nanoparticles with Nano-Localized Hyperpyrexia
}

Dengfeng Hu, Lingyun Zou, Bochao Li, Mi Hu, Wanying Ye and Jian Ji*

MOE Key Laboratory of Macromolecule Synthesis and Functionalization of Ministry of Education, Department of Polymer Science and Engineering, Zhejiang University, Hangzhou, 310027, China.

*Corresponding author

*E-mail: jijian@,zju.edu.cn (Jian Ji)

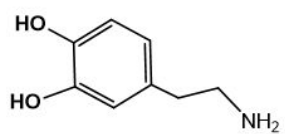

oxidative and alkaline conditions

dopamine

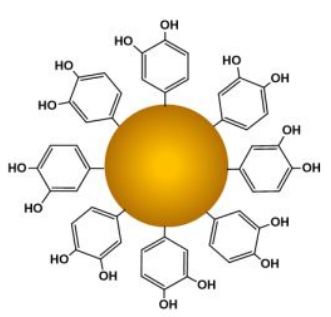

polydopamine (PDA)

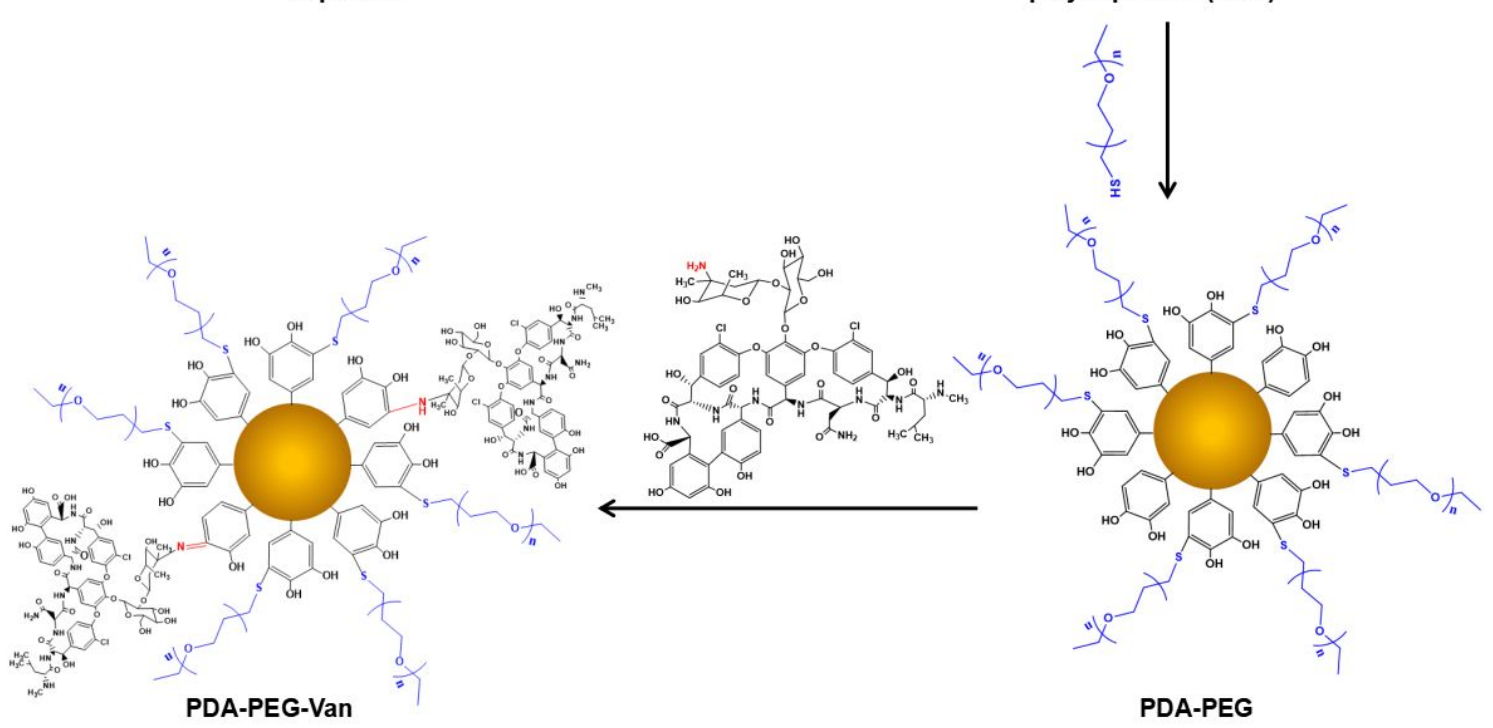


Figure S1. Detailed synthetic routes of PDA-PEG and PDA-PEG-Van nanoparticles.
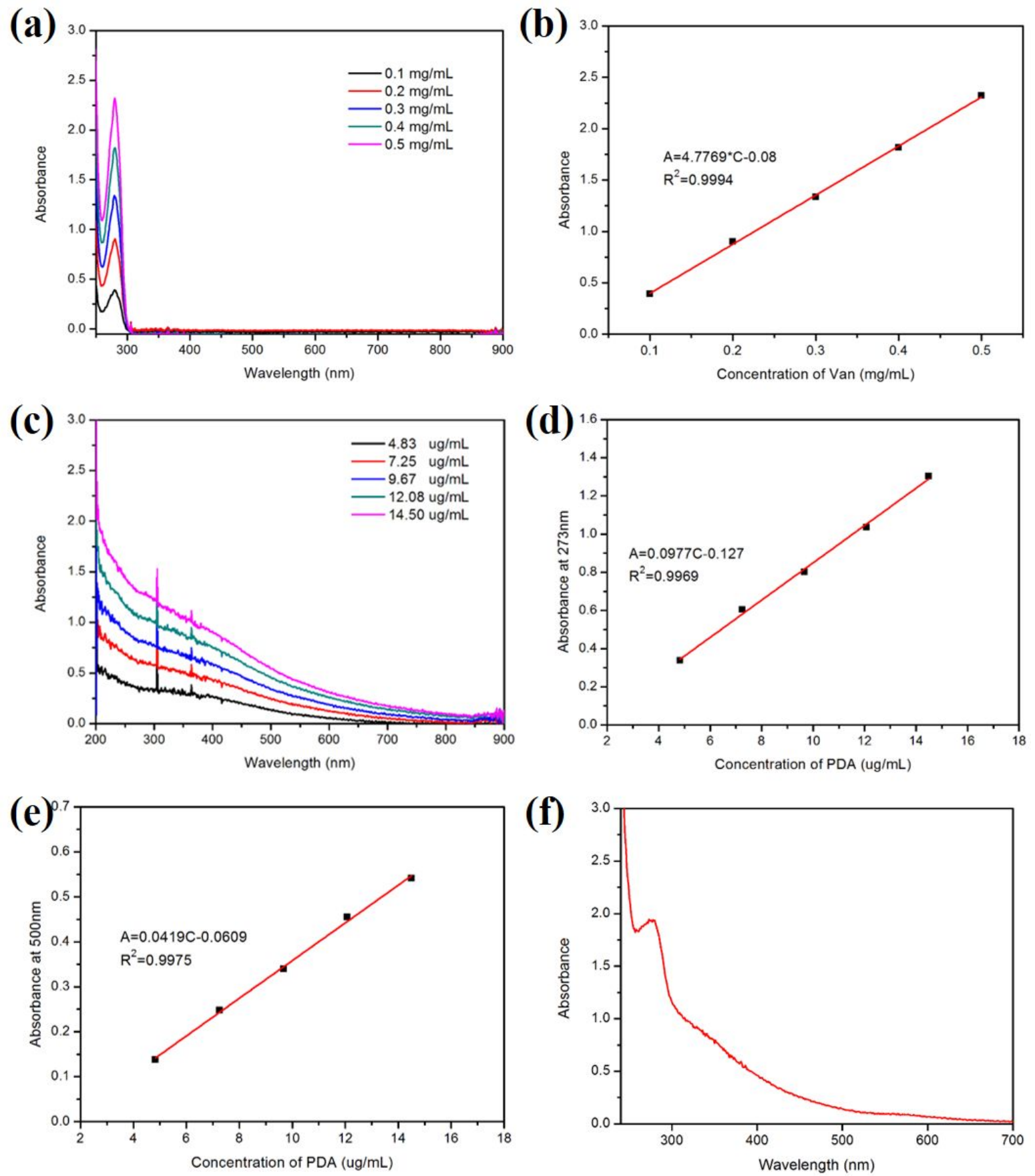

Figure S2. (a) UV-Vis absorption spectra of Van molecules with various concentrations; (b) The linear calibration curve between Van concentration and its absorbance at $273 \mathrm{~nm}$; (c) UVVis absorption spectra of PDA-PEG with various concentrations; (d) The linear calibration curve between PDA-PEG concentration and its absorbance at $273 \mathrm{~nm}$; (e) The linear calibration curve between PDA-PEG concentration and its absorbance at $500 \mathrm{~nm}$; (f) UV-Vis absorption spectra of the Van in the supernatant after centrifugation. 
PDA
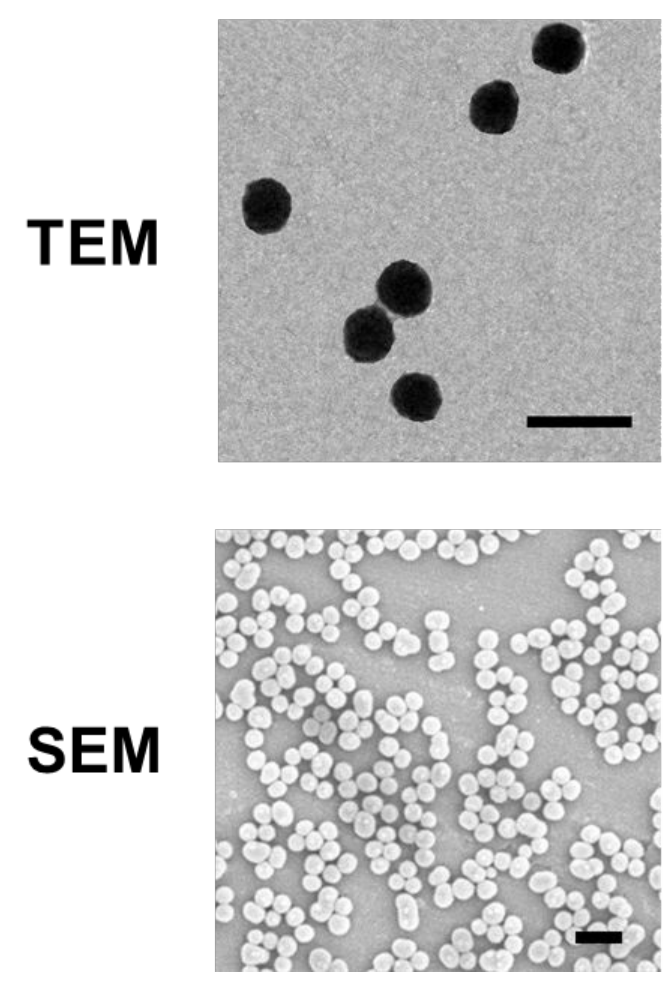

PDA-PEG
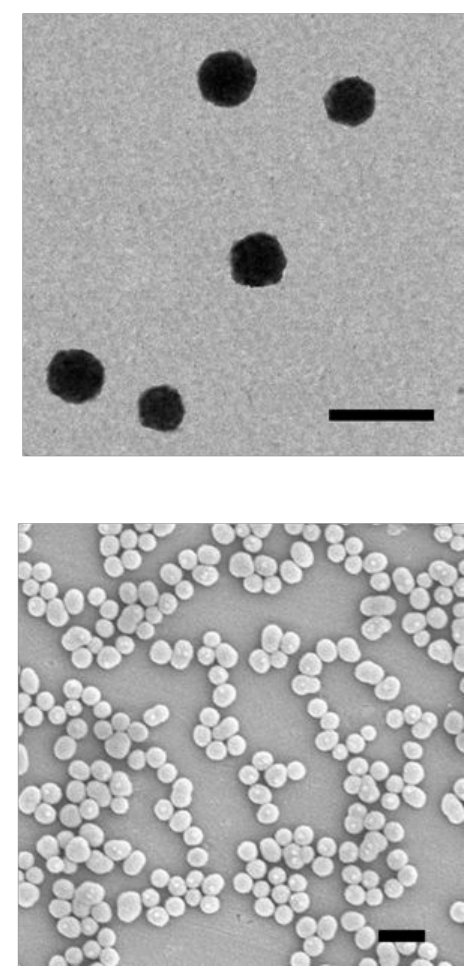

Figure S3. TEM and SEM images of PDA and PDA-PEG in phosphate buffered saline (PBS, $10 \mathrm{mM}, \mathrm{pH} 7.4)$, scale bar is $200 \mathrm{~nm}$.

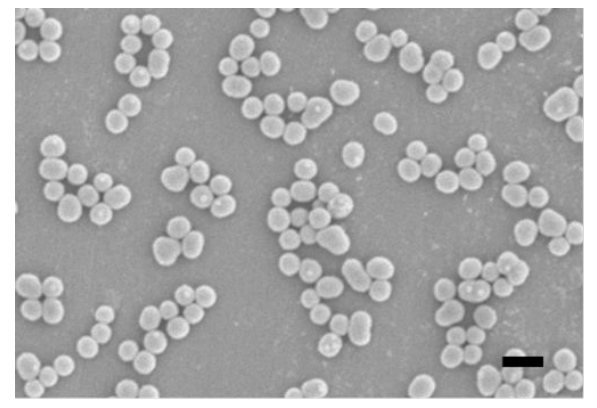

Before NIR irradiation

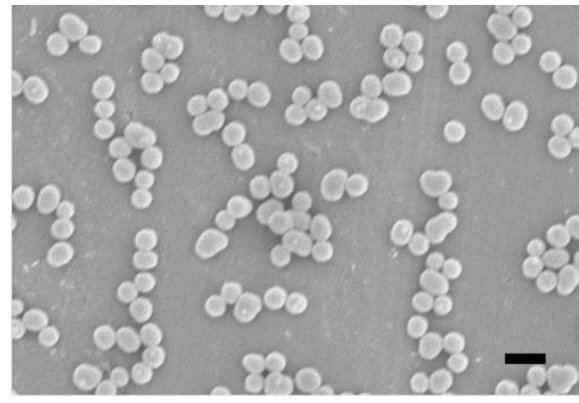

After NIR irradiation

Figure S4. Zeta potentials of PDA, PDA-PEG and PDA-PEG-Van in phosphate-buffered saline (PBS) buffer (10 mM, pH 7.4), scale bar is $200 \mathrm{~nm}$. 